\title{
Noise-based approximation to thermal spin-injection in Fe/GaAs
}

\author{
T. Wagner, ${ }^{1,2}$ J. A. Haigh, ${ }^{1}$ K. Olejník, ${ }^{3}$ A. C. Irvine, ${ }^{4}$ V. Novák, ${ }^{3}$ and J. Wunderlich ${ }^{1,3}$ \\ ${ }^{1)}$ Hitachi Cambridge Laboratory, Cambridge, CB3 OHE, UK \\ ${ }^{2)}$ Department of Materials Science and Metallurgy, University of Cambridge, Cambridge, CB3 OFS, \\ $U K$ \\ ${ }^{3)}$ Institute of Physics ASCR, v.v.i., Cukrovarnická 10, 16253 Praha 6, Czech Republic \\ ${ }^{4)}$ Cavendish Laboratory, University of Cambridge, Cambridge, CB3 OHE, UK
}

(Dated: September 12, 2017)

We analyze the prospects for thermal spin injection from iron into gallium arsenide via the application of electrical noise. By estimating the applied effective temperature-equivalent gradients, we characterize the magnitude of any electrical part of the thermal spin injection efficiency, or spin-dependent Seebeck effect. The magnitude of the nonlocal spin signal associated with this effect suggests that temperature differences of the order of $\sim 100 \mathrm{~K}$ would be needed for true thermal spin injection experiments. The large size of the effective temperature gradients induced by the noise-based method means that even very small thermo-electric effects can be quantified.

The creation and detection of non-equilibrium spin polarization through electrical contacts of ferromagnetic to nonmagnetic materials has been comprehensively studied in both metals $^{1-3}$ and semiconductors ${ }^{4-6}$. This has led to new insight into the dynamics of non-equilibrium spin polarizations, including the spin Hall effect ${ }^{7-9}$, nuclear spin polarization ${ }^{10-12}$ and the effect of spin-orbit fields ${ }^{13}$.

Whilst typically electrical bias is used to drive spinpolarized charge carriers from the ferromagnet into the nonmagnetic materials, more recently temperature differences have been applied across this interface in order to study the spin dependence of thermoelectric effects. The resulting thermal-spin-injection has been measured in metallic devices ${ }^{14}$, silicon, ${ }^{15}$ and germanium ${ }^{16,17}$ and is discussed in terms of a spin-dependent Seebeck effect or spin Seebeck tunneling associated with the interface ${ }^{15,17}$. However, thermal spin injection has yet to be demonstrated in the archetypal metal-semiconductor electrical spin-injection system, Fe/GaAs ${ }^{4}$. The formation of a Schottky barrier at the $\mathrm{Fe} / \mathrm{GaAs}$ interface eliminates the conductivity mismatch, and all-electrical spin injection and detection is routinely studied in non-local spin valve devices. Attempts to observe thermal spin-injection in this system may have gone unpublished as it is difficult to quantify any lack of signal due to the reliance on finite element modeling in estimating temperature differences across the nm-scale tunnel barrier ${ }^{17}$. This makes it hard to rule out any effect based on direct thermal measurements.

Instead, in this letter we attempt to characterize the prospects for thermal spin injection in Fe/GaAs by creating artificially large effective temperature differences through the application of electrical noise. This allows us to estimate the magnitude of the electrical contribution to any thermal spininjection, even though the effect is likely too small to be measurable directly. This strictly is not thermal spin-injection: in particular, the noise does not raise the effective temperature of either the phonon or magnon baths, which may contribute to the spin-injection through phonon drag and spin-pumping effects, respectively. However, it may offer an indication as to why the effect has not been observed in this particular system. The technique we demonstrate may also be applied in other systems in discriminating between the different contributions to thermal spin-injection.
The relationship between voltage fluctuations and temperature is well known, and is most directly demonstrated in the Johnson-Nyquist formula for the equilibrium voltage noise across a resistor ${ }^{18,19}$, a manifestation of the more widely applicable fluctuation-dissipation relation ${ }^{20}$. Away from equilibrium, thermally driven transport has been analyzed in terms of noise-induced currents ${ }^{21,22}$ and experimentally, fluctuations applied to the gate of a nano-electronic device have been used to characterize its thermoelectric performance ${ }^{23}$. Different types of noise and their relation to thermoelectric transport coefficients was discussed in a recent theoretical study ${ }^{24}$. Here, we use a simple argument based on the Landauer-Büttiker transport formalism to relate the equilibrium distribution functions at an effective temperature to an effective distribution function with applied voltage fluctuations. This relation allows us to estimate the Seebeck-like transport coefficients for our noise-based approximation to thermal spin-injection. As shown in Fig. 1 voltage fluctuations and temperature differences similarly probe differences in density of states above and below the electrochemical potential. In some sense this is an application of the Mott formula ${ }^{25}$, but instead of estimating the energy dependence of the conductance, for example from $\mathrm{d} I / \mathrm{d} V$ measurements ${ }^{26}$, we use that relation to find a connection between the response to applied voltage fluctuations and the thermoelectric effects.

Before proceeding to the experimental results we outline the proposed connection between thermoelectric Seebeck effect and response to applied voltage fluctuations. We limit ourselves to discussion of the normal Seebeck effect before extending the analysis to the spin-injection analogue.

As the tunneling at the interface is expected to dominate any thermal spin injection ${ }^{15}$, we are interested in the transport properties of the tunnel junction at the Fe/GaAs interface due to the Schottky barrier. The standard expression for calculating the current between two electronic systems separated by a tunnel barrier can be written as an energy $E$ integral $^{27}$

$$
\begin{aligned}
I=-\frac{2 e}{h} \int_{-\infty}^{\infty} & D_{\mathrm{l}}\left(E-\mu_{\mathrm{l}}\right) \mathcal{T}(E-\bar{\mu}) D_{\mathrm{r}}\left(E-\mu_{\mathrm{r}}\right) \\
& \times\left[f_{\mathrm{l}}\left(E-\mu_{\mathrm{l}}\right)-f_{\mathrm{r}}\left(E-\mu_{\mathrm{r}}\right)\right] \mathrm{d} E
\end{aligned}
$$

where $e$ is the elementary charge, $h$ is Planck's constant, $D$ 
is the density of states, $\mathcal{T}$ is the transmission through the barrier, $\mu$ is the electrochemical potential and the subscript $1(\mathrm{r})$ specifies the left (right) electrode. The mean electrochemical potential of the electrodes is $\bar{\mu}=\left(\mu_{1}+\mu_{\mathrm{r}}\right) / 2$, which in case of a symmetric voltage drop across the barrier is the equilibrium electrochemical potential. In the usual cases treated by the Landauer-Büttiker formalism the distribution function $f$ is equal to the Fermi-Dirac distribution,

$$
\mathfrak{f}(E)=\frac{1}{e^{\left(\frac{E-\mu}{k_{\mathrm{B}} T}\right)}+1},
$$

where $T$ is the temperature and $k_{\mathrm{B}}$ is Boltzmann's constant.

Instead of establishing a temperature difference between the two electrodes (the Seebeck effect in Fig. 1(a)), we consider a fluctuating voltage applied across the barrier with a Gaussian probability distribution function (Fig. 1(b)) defined by the standard deviation $\sigma$ applied around $\bar{\mu}$,

$$
P(\mu)=\frac{1}{\sqrt{2 \sigma^{2} \pi}} e^{-\frac{(\mu-\bar{\mu})^{2}}{2 \sigma^{2}}} .
$$

When the Gaussian probability distribution is applied to a system with Fermi-Dirac distribution $\mathfrak{f}$, the resulting distribution is given by a convolution of the individual distributions: $\bar{f}=P * \mathfrak{f}$. In order to study the effect of the voltage fluctuations on the right electrode we assume that the density of states of the counterelectrode $D_{1}$ and the transmission function $\mathcal{T}$ of the tunnel junction are both independent of energy around the electrochemical potential. With the altered distribution function

$$
\overline{f_{1}}(E, \bar{\mu})=\int_{-\infty}^{\infty} P(\mu) \mathfrak{f}_{1}(E, \mu) \mathrm{d} \mu
$$

of the left electrode we find the time-averaged electric current from Eq. (1)

$$
\bar{I}=-\frac{2 e}{h} \int_{-\infty}^{\infty} D_{\mathrm{l}} \mathcal{T} D_{\mathrm{r}}(E)\left[\bar{f}_{1}(E)-f_{\mathrm{r}}(E)\right] \mathrm{d} E .
$$

The conductance of the junction, taking into account the energy dependence of density of states of the right electrode, can be written as $G(E)=\frac{2 e^{2}}{h} D_{1} \mathcal{T} D_{\mathrm{r}}(E)$. We proceed from Eq. (1) with a Sommerfeld-type expansion ${ }^{28}$ to approximate the integral giving the current. Following Ref. 29, we obtain

$$
\begin{aligned}
I=-\frac{1}{e} & {\left[\int_{-\infty}^{\mu_{1}} G(E) \mathrm{d} E+\left.\sum_{n=1}^{\infty} m_{2 n}^{1} \frac{1}{(2 n) !} \frac{\mathrm{d}^{2 n-1} G(E)}{\mathrm{d} E^{2 n-1}}\right|_{\mu_{1}}\right.} \\
& \left.-\int_{-\infty}^{\mu_{\mathrm{r}}} G(E) \mathrm{d} E-\left.\sum_{n=1}^{\infty} m_{2 n}^{\mathrm{r}} \frac{1}{(2 n) !} \frac{\mathrm{d}^{2 n-1} G(E)}{\mathrm{d} E^{2 n-1}}\right|_{\mu_{\mathrm{r}}}\right]
\end{aligned}
$$

where $m_{2 n}^{1(r)}$ is the $2 n$th moment of the derivative of the distribution function in the left (right) lead with respect to energy,

$$
m_{2 n}^{1(\mathrm{r})}=\int_{-\infty}^{\infty}\left(E-\mu_{1(\mathrm{r})}\right)^{2 n}\left(-\frac{\partial f_{1(\mathrm{r})}}{\partial E}\right) \mathrm{d} E .
$$

a)
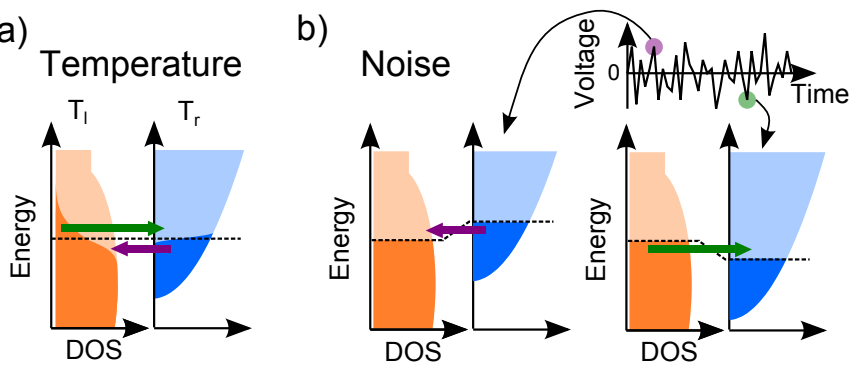

Figure 1. (a) The current in the standard Seebeck effect of a tunnel junction is driven between two electrodes at temperatures $T_{1}$ and $T_{\mathrm{r}}$, separated by a barrier. Differences in electron flow below (purple arrow) and above (green arrow) the electrochemical potential (dashed black line) contribute to the net current. (b) Seebeck-like effect with Gaussian voltage fluctuations applied to the tunnel junction. The time-averaged current can be decomposed into contributions at different times. Positive and negative voltages probe the density of states in a similar way to the Seebeck effect.

This assumes that the derivative of the distribution function is only significant in some region around the chemical potential, about which it is symmetric ${ }^{29}$. Eq. (6) can be rewritten in terms of differences in the electrochemical potentials $\Delta \mu=\mu_{1}-\mu_{\mathrm{r}}$ and differences in the moments of the distribution functions $\Delta m_{2 n}=m_{2 n}^{1}-m_{2 n}^{\mathrm{r}}$,

$$
\begin{aligned}
I \approx & -\frac{1}{e} \Delta \mu G(\bar{\mu}) \\
& -\left.\frac{1}{e} \sum_{n=1}^{\infty} \Delta m_{2 n} \frac{1}{(2 n) !} \frac{\mathrm{d}^{2 n-1} G(E)}{\mathrm{d} E^{2 n-1}}\right|_{\bar{\mu}} .
\end{aligned}
$$

Here, the first term corresponds to electrically driven current, while the Seebeck effect appears in the second term. For $f_{\mathrm{r}}(E)=\mathfrak{f}(E)$ and keeping only the first term in the summation, for which $m_{2}^{1(\mathrm{r})}=\frac{\pi^{2}}{3}\left(k_{\mathrm{B}} T_{1(\mathrm{r})}\right)^{2}$, the current reduces to the expected result

$$
I=-\left.\frac{\pi^{2}{k_{\mathrm{B}}}^{2}}{3 e} \bar{T} \Delta T \frac{\mathrm{d} G(E)}{\mathrm{d} E}\right|_{\bar{\mu}},
$$

where $\bar{T}=\left(T_{1}+T_{\mathrm{r}}\right) / 2$, and $\Delta T=T_{1}-T_{\mathrm{r}}$.

Next, by evaluating the second moment of the derivative of the effective distribution function we can compare the thermoelectric current to the noise induced effects in our devices. The derivative of the effective distribution function is

$$
\frac{\partial \bar{f}}{\partial E}=\frac{\partial}{\partial E}(P * \mathfrak{f})=P * \frac{\partial \mathfrak{f}}{\partial E},
$$

the second moment of which is simply the sum of those of the individual functions, $\sigma^{2}$ and $\frac{\pi^{2}}{3}\left(k_{\mathrm{B}} T\right)^{2}$ respectively ${ }^{30}$. This allows us to find a correspondence between the applied noise and temperature, with the effective temperature of the electrodes given by

$$
T_{\text {eff }}=\sqrt{T^{2}+3 \sigma^{2} / \pi^{2} k_{\mathrm{B}}^{2}} .
$$


a) i)

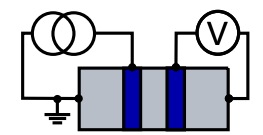

ii)

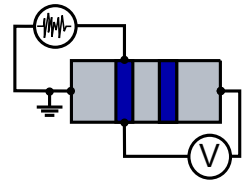

iii)
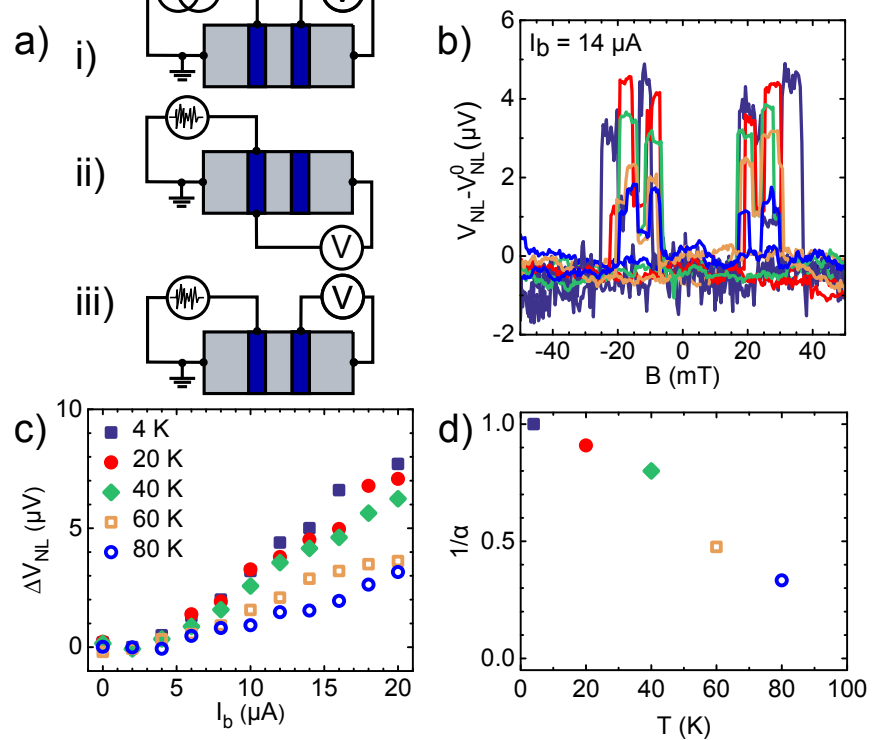

Figure 2. (a) Connections to the non-local spin valve device for different experiments (light grey: GaAs, dark blue: Fe). (i) For standard electrical spin-injection a current is applied to the injection contact, and the non-local voltage measured on the detection contact. (ii,iii) For noise measurements an arbitrary waveform generator is used to apply Gaussian white noise across the injection contact of the device. We then measure either the local (ii) or non-local voltage (iii) on the injector or detector contact respectively. (b) Non-local spinvalve measurements demonstrating electrical spin-injection at different temperatures. (c) Injection current dependence of spin-valve signal at different temperatures. (d) Temperature dependence of the effective detection sensitivity.

The Seebeck effect is quantified by the usual Seebeck coefficient, which relates the open circuit voltage to the applied temperature difference $V=-S \Delta T$. From the Eq. (8), the open circuit voltage is

$$
V=-\frac{I}{G(\bar{\mu})}=\left.\Delta T \bar{T} \frac{\pi^{2} k_{\mathrm{B}}^{2}}{3 e} \frac{1}{G(\bar{\mu})} \frac{\mathrm{d} G(E)}{\mathrm{d} E}\right|_{\bar{\mu}},
$$

from which the standard expression for the Seebeck coefficient is found

$$
S=-\left.\bar{T} \frac{\pi^{2} k_{\mathrm{B}}^{2}}{3 e} \frac{1}{G(\bar{\mu})} \frac{\mathrm{d} G(E)}{\mathrm{d} E}\right|_{\bar{\mu}} .
$$

We will use these standard expressions, but with the effective temperatures given by Eq. (11) as the arguments, to analyze our measurements.

The devices used for experiments were fabricated from a $\mathrm{Fe} / \mathrm{GaAs}$ heterostructure grown by molecular beam epitaxy $4,9,10,12,31$. The active semiconductor layer consists of a $250 \mathrm{~nm}$ thick low Si-doped GaAs $\left(n_{\mathrm{Si}}=5 \times 10^{16} \mathrm{~cm}^{-3}\right)$ transport channel followed by a $15 \mathrm{~nm}$ thick layer of graded doping and $15 \mathrm{~nm}$ of highly Si-doped GaAs $\left(n_{\mathrm{Si}}=6 \times 10^{18} \mathrm{~cm}^{-3}\right)$. A $2 \mathrm{~nm}$ layer of Fe was grown on top without breaking the vacuum and capped by $3 \mathrm{~nm}$ of $\mathrm{Al}$ to prevent oxidation of the ferromagnet. A narrow Schottky barrier is formed in the $\mathrm{GaAs}^{4}$ with barrier height around $0.2-0.8 \mathrm{~V}^{32}$ and thickness $\sim 3 \mathrm{~nm}$.
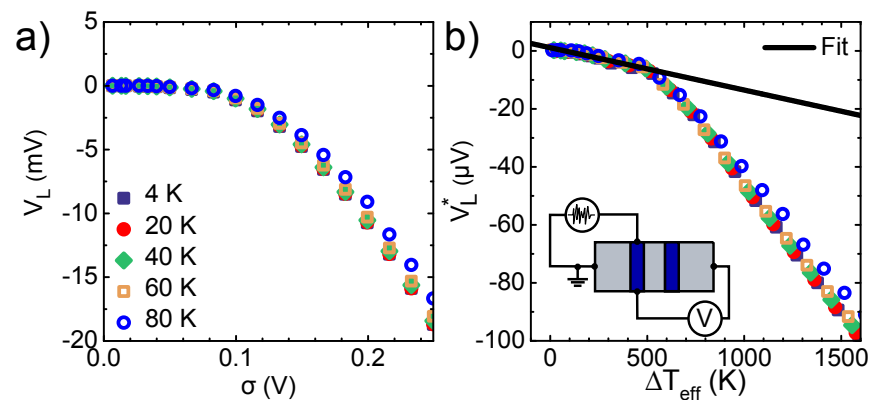

Figure 3. Noise-driven local Seebeck-like voltages. (a) Measured dc voltages plotted as a function of the standard deviation of the applied voltage noise for various temperatures. (b) Rescaled data to extract the Seebeck coefficient. The effective temperature difference is calculated from the standard deviation of the noise via Eq. (11) and the voltages are rescaled by $\bar{T} / T_{\text {ref. The linear fit to the initial data points }}$ gives an effective Seebeck coefficient $-15 \mathrm{nVK}^{-1}$.

The sample was patterned into non-local spin valve devices by optical and electron beam lithography. Wet chemical etching down to the intrinsic layer was used to define a bar structure with a $20 \mu \mathrm{m}$ wide transport channel along the [110] crystal direction of GaAs. The Fe injector and detector electrodes $\left(1 \times 19 \mu \mathrm{m}^{2}\right)$, patterned by metal evaporation and subsequent wet chemical etching, were aligned with their long axis in the [110] crystal direction of GaAs. The surface-induced magneto-crystalline anisotropy of the ultrathin $\mathrm{Fe}$ layer ${ }^{33}$ together with the lithographically imposed shape anisotropy makes the long axis of the $\mathrm{Fe}$ contacts the magnetic easy axis. Finally, $\mathrm{Cr} / \mathrm{Au}$ lines were evaporated to contact the Fe stripes supported by cross-linked PMMA at the sides of the GaAs bar to be electrically insulated from the doped layers. From the five Fe contacts per bar, with centerto-center spacings of $2 \mu \mathrm{m}$, a neighboring pair was chosen for non-local spin valve experiments.

Measurements were made in a Helium flow cryostat between $4.2 \mathrm{~K}$ and $80 \mathrm{~K}$. In all experiments presented the magnetic field is applied along the magnetic easy axis to switch the Fe contacts in a controlled manner.

We first demonstrate standard electrical spin-injection by measuring the non-local voltage on the detector contact as an electrical current is driven through the injection contact (Fig. 2(a)(i)). Non-local spin-valve measurements are shown in Fig. 2(b) for various temperatures. We can clearly resolve the signal up to $80 \mathrm{~K}$. In Fig. 2(c) the measured amplitude of the electrical spin signal is plotted as a function of injection current for these temperatures. The change is consistent with measurements by others ${ }^{34,35}$ in which the spin-signal is largely dominated by spin decay during transport through the non-magnetic channel. We quantify this decay in detection efficiency by the scaling factor $\alpha=\Delta V_{\mathrm{NL}}(T) / \Delta V_{\mathrm{NL}}(4.2 \mathrm{~K})$ independent of the bias current. This is plotted in Fig. 2(d) and will be important later as it calibrates the temperature dependence of the detection sensitivity, allowing us to compare injection efficiency of any thermal-like spin-injection at different temperatures.

Next we apply the noise source to the injector contact 
(Fig. 2(a)(ii)). The noise is spectrally flat with a bandwidth $B \approx 1.9 \mathrm{MHz}$, and the standard deviation is directly measured on the contact from fitting the distribution of voltages recorded on an oscilloscope to account for attenuation in the cryostat wiring and contact resistances. We then use Eq. (11) to equate this with an effective temperature. In order to accurately measure the small noise-induced voltages, we amplitude modulate the applied noise and measure the dc voltages with a lock-in amplifier.

A voltage applied to a tunnel junction is, by definition, the difference in electrical potential between the left and the right electrode. Therefore, the noise voltage can be regarded to drop symmetrically across the barrier and it is not a priori clear for which of the electrodes heating is simulated. However, the electrode with an energy-independent density of states receives a net flow of electrons from below the electrochemical potential of the other electrode for one voltage polarity and injects electrons into states above the electrochemical potential for the opposite polarity. Therefore, the electrode with the energy-independent density of states resembles the hot electrode in a classical Seebeck measurement. The dc voltage drop detected in the measurement is referenced to a specific electrode, in this case the GaAs transport channel, so that the noise-Seebeck voltage is always measured relative to the potential of this electrode.

The local dc voltages resulting from this noise are plotted in Fig. 3(a). This corresponds to the normal Seebeck effect of the tunnel junction ${ }^{36}$. At the low temperatures at which we measure, $T_{\text {eff }}$ varies significantly as the standard deviation of the noise is varied. From Eqn. 13 the characteristic linear temperature dependence of the Seebeck effect can be seen. In order to recover this linear behavior we normalize all voltages by $\bar{T}$ and project all measurements to $T_{\text {ref }}=4.2 \mathrm{~K}$. This is shown in Fig. 3(b). All the measurements at different temperatures lie on a single line up to $\Delta T_{\text {eff }} \approx 500 \mathrm{~K}$, below which a linear fit gives an effective Seebeck coefficient at $4.2 \mathrm{~K}$ of $-15 \mathrm{nVK}^{-1}$. For comparison, the Seebeck coefficient of $\mathrm{Fe}$ at $4.2 \mathrm{~K}$ is $\sim 200 \mathrm{nVK}^{-137}$, dominated by magnon-drag ${ }^{38}$. We emphasize that because the bandwidth of the applied noise is lower than typical magnon frequencies, we are not sensitive to magnon-drag effects. When the applied voltage fluctuations become too large, the measured data no longer follows the expected behavior, likely because the assumptions in our analysis break down: the energy-dependence of the transmission $\mathcal{T}$ and of the density of states $D_{1}$ of the Fe electrode can no longer be neglected.

Now we apply the same procedure to the non-local voltage (Fig. 2(a)(iii)). The results are shown in Fig.4(a). The measurements are complicated by the change in the spin diffusion length and spin detection efficiency of the detector electrode with temperature, which we have to account for in order to compare data at different temperatures. To do this the extracted scaling coefficient $\alpha$ from Fig. 2(d) is used to map the measured noise induced non-local voltages to the same detection sensitivity as at $4.2 \mathrm{~K}$. When the non-local spindependent Seebeck-like voltages are normalized in this way the results coincide on a single curve up to $\Delta T_{\text {eff }} \approx 500 \mathrm{~K}$ as depicted in Fig. 4(b), from which we extract the effective
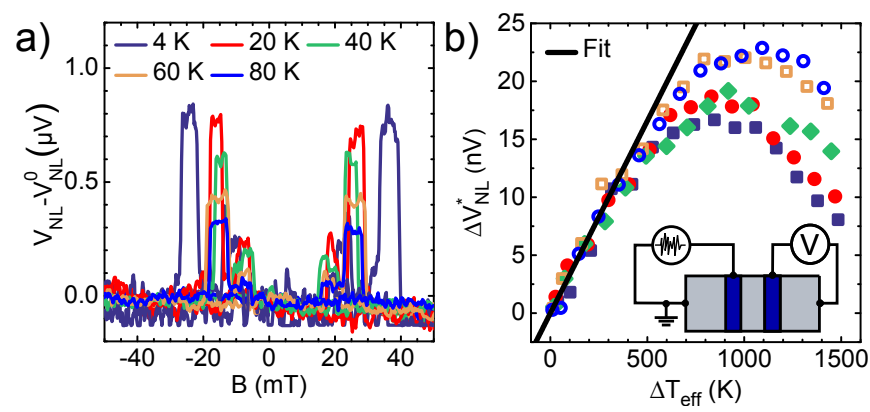

Figure 4. (a) Noise-driven non-local spin-valve measurements. The non-local voltage measured as a function of magnetic field at different temperatures for $\sigma=59 \mathrm{mV}$. The non-local Seebeck voltages are extracted from the height of the switched region. (b) Non-local Seebeck-like voltages, rescaled to $T_{\text {ref }}$ using the factor $\alpha \bar{T} / T$. The linear fit to the initial data gives an effective non-local Seebeck coefficient of $33 \mathrm{pV} \mathrm{K}^{-1}$.

non-local spin-dependent Seebeck coefficient as $33 \mathrm{pV} \mathrm{K}^{-1}$.

In our spin detection experiments we are sensitive to a change in the non-local voltage of $\sim 100 \mathrm{nV}$, which in case of thermal spin injection would require a temperature difference of the order of $100 \mathrm{~K}$ across the barrier. This can be compared to the results of a temperature profile calculation in a simple finite element model of our device ${ }^{14,39}$. The model uses a Gaussian-shaped heat source in the top gold layer of the injector contact to simulate heating by a focused laser (beam diameter $2 \mu \mathrm{m}$ ) with exponential decay into the sample (the absorption length was taken as $14 \mathrm{~nm}$, calculated from the optical constants in gold at a wavelength of $650 \mathrm{~nm}^{40}$ ). The temperature difference is evaluated as $\approx 5 \mathrm{mK}$ per absorbed $\mathrm{mW}$ of laser power for a distance of $15 \mathrm{~nm}$, which is the thickness of the highly doped GaAs layer where the Schottky barrier is formed. It should be noted that, depending on laser wavelength, the actual laser power required to generate this amount of heating can be much larger due to the large reflection coefficient of the $\mathrm{Au} /$ vacuum interface. From this rough estimate it seems unlikely the required temperature differences could be achieved in a cw-optical heating experiment.

Additionally, the effective temperature mimicked by voltage noise corresponds only to the electron system; the magnon temperature of the ferromagnetic electrode is not raised, nor is the phonon temperature. This means that possible spin-injection due to spin-pumping ${ }^{41}$ or phonon-drag ${ }^{42,43}$ effects could give contributions that would allow thermal spininjection to be observable at reasonable temperature differences. We note that with a larger bandwidth $(>\mathrm{GHz})$ noise source, it may be possible to use a similar technique to study the thermal analog of spin-pumping, with incoherent instead of coherent driving of the magnetization precession.

In summary we have attempted to address the possibility of thermal spin-injection from iron into gallium arsenide via the application of noise voltages to the injection contact. By making a comparison in the broadening of the Fermi-Dirac distribution for real temperatures and applied voltage fluctuations, we estimate the thermal spin-injection efficiency. From this estimation, it seems experimentally unfeasible to measure 
actual steady-state thermal spin-injection from heating of the ferromagnet's electron system alone. This may explain why, while thermal spin-injection has been demonstrated in some semiconductor systems, it has not been identified in Fe/GaAs. In systems where thermal spin injection has been observed, our technique may be helpful in confirming that electronic contribution is dominant over phonon and magnon driven processes. In addition, the much larger effective temperature differences that it is possible to apply with our noise-based technique allow even very small effects to be quantified.

\section{ACKNOWLEDGMENTS}

We thank A. Crépieux and G. Schmidt for helpful discussions. The research leading to these results has received funding from the European Union Seventh Framework Programme ([FP7/2007-2013] [FP7/2007-2011]) under grant agreement 316657 (SpinIcur). J.W. acknowledges support from the European Research Council Synergy Grant No. 610115.

\section{REFERENCES}

${ }^{1}$ M. Johnson and R. H. Silsbee, Phys. Rev. Lett. 55, 1790 (1985).

${ }^{2}$ M. Johnson and R. H. Silsbee, Phys. Rev. B 37, 5326 (1988).

${ }^{3}$ F. J. Jedema, A. T. Filip, and B. J. van Wees, Nature 410, 345 (2001).

${ }^{4}$ X. Lou, C. Adelmann, S. A. Crooker, E. S. Garlid, J. Zhang, K. S. M. Reddy, S. D. Flexner, C. J. Palmstrøm, and P. A. Crowell, Nat Phys 3, 197 (2007).

${ }^{5}$ I. Appelbaum, B. Huang, and D. J. Monsma, Nature 447, 295 (2007).

${ }^{6}$ M. Ciorga, A. Einwanger, U. Wurstbauer, D. Schuh, W. Wegscheider, and D. Weiss, Phys. Rev. B 79, 165321 (2009).

${ }^{7}$ S. O. Valenzuela and M. Tinkham, Nature 442, 176 (2006).

${ }^{8}$ E. S. Garlid, Q. O. Hu, M. K. Chan, C. J. Palmstrøm, and P. A. Crowell, Phys. Rev. Lett. 105, 156602 (2010).

${ }^{9}$ K. Olejník, J. Wunderlich, A. C. Irvine, R. P. Campion, V. P. Amin, J. Sinova, and T. Jungwirth, Phys. Rev. Lett. 109, 076601 (2012).

${ }^{10}$ G. Salis, A. Fuhrer, and S. F. Alvarado, Phys. Rev. B 80, 115332 (2009).

${ }^{11}$ M. K. Chan, Q. O. Hu, J. Zhang, T. Kondo, C. J. Palmstrøm, and P. A. Crowell, Phys. Rev. B 80, 161206 (2009).

${ }^{12}$ C. Awo-Affouda, O. M. J. van 't Erve, G. Kioseoglou, A. T. Hanbicki, M. Holub, C. H. Li, and B. T. Jonker, Applied Physics Letters 94, 102511 (2009).

${ }^{13}$ H. C. Koo, J. H. Kwon, J. Eom, J. Chang, S. H. Han, and M. Johnson, Science 325, 1515 (2009).

${ }^{14}$ A. Slachter, F. L. Bakker, J.-P. Adam, and B. J. van Wees, Nat Phys 6, 879 (2010).

${ }^{15}$ J.-C. Le Breton, S. Sharma, H. Saito, S. Yuasa, and R. Jansen, Nature 475, 82 (2011).

${ }^{16}$ A. Jain, C. Vergnaud, J. Peiro, J. C. Le Breton, E. Prestat, L. Louahadj, C. Portemont, C. Ducruet, V. Baltz, A. Marty, A. Barski, P. BayleGuillemaud, L. Vila, J.-P. Attané, E. Augendre, H. Jaffrès, J.-M. George, and M. Jamet, Applied Physics Letters 101, 022402 (2012).

${ }^{17}$ K.-R. Jeon, B.-C. Min, S.-Y. Park, K.-D. Lee, H.-S. Song, Y.-H. Park, Y.-H. Jo, and S.-C. Shin, Scientific Reports 2, 962 (2012).

${ }^{18}$ J. B. Johnson, Phys. Rev. 32, 97 (1928).

${ }^{19}$ H. Nyquist, Phys. Rev. 32, 110 (1928).

${ }^{20}$ R. Kubo, Reports on Progress in Physics 29, 306 (1966).

${ }^{21}$ M. Büttiker, Z. Physik B - Condensed Matter 68, 161 (1987).

${ }^{22}$ R. Landauer, J Stat Phys 53, 233 (1988).

${ }^{23}$ F. Hartmann, P. Pfeffer, S. Höfling, M. Kamp, and L. Worschech, Phys. Rev. Lett. 114, 146805 (2015).

${ }^{24}$ A. Crépieux and F. Michelini, Journal of Physics: Condensed Matter 27, 1 (2015).
${ }^{25}$ M. Cutler and N. F. Mott, Phys. Rev. 181, 1336 (1969).

${ }^{26}$ R. Meservey and P. M. Tedrow, Physics Reports 238, 173 (1994).

${ }^{27}$ T. Ihn, Semiconductor Nanostructures: Quantum states and electronic transport (Oxford University Press, Oxford, 2009).

${ }^{28}$ A. Sommerfeld, Z. Physik 47, 1 (1928).

${ }^{29}$ N. W. Ashcroft and N. Mermin, Solid State Physics (Brooks/Cole, New York, 1976).

${ }^{30}$ E. T. Jaynes, Probability Theory: The Logic of Science, edited by G. L. Bretthorst (Cambridge University Press, Cambridge, 2003).

${ }^{31}$ M. K. Chan, Q. O. Hu, J. Zhang, T. Kondo, C. J. Palmstrøm, and P. A. Crowell, Phys. Rev. B 80, 161206 (2009).

${ }^{32}$ L. R. Fleet, K. Yoshida, H. Kobayashi, Y. Ohno, H. Kurebayashi, J. Y. Kim, C. H. W. Barnes, and A. Hirohata, IEEE Transactions on Magnetics 46, 1737 (2010).

${ }^{33}$ G. Wastlbauer and J. A. C. Bland, Advances in Physics 54, 137 (2005).

${ }^{34}$ G. Salis, R. Wang, X. Jiang, R. M. Shelby, S. S. P. Parkin, S. R. Bank, and J. S. Harris, Applied Physics Letters 87, 262503 (2005).

${ }^{35}$ G. Salis, A. Fuhrer, R. R. Schlittler, L. Gross, and S. F. Alvarado, Phys. Rev. B 81, 205323 (2010).

${ }^{36}$ A. D. Smith, M. Tinkham, and W. J. Skocpol, Phys. Rev. B 22, 4346 (1980).

${ }^{37}$ S. J. Watzman, R. A. Duine, Y. Tserkovnyak, S. R. Boona, H. Jin, A. Prakash, Y. Zheng, and J. P. Heremans, Phys. Rev. B 94, 1 (2016).

${ }^{38}$ F. J. Blatt, D. J. Flood, V. Rowe, P. A. Schroeder, and J. E. Cox, Phys. Rev. Lett. 18, 395 (1967).

${ }^{39}$ COMSOL, COMSOL Multiphysics.

${ }^{40}$ P. B. Johnson and R. W. Christy, Phys. Rev. B 6, 4370 (1972).

${ }^{41}$ K. Ando, S. Takahashi, J. Ieda, H. Kurebayashi, T. Trypiniotis, C. H. W. Barnes, S. Maekawa, and E. Saitoh, Nat Mater 10, 655 (2011).

${ }^{42}$ H. Adachi, K. Uchida, E. Saitoh, J. Ohe, S. Takahashi, and S. Maekawa, Applied Physics Letters 97, 252506 (2010).

${ }^{43}$ C. M. Jaworski, J. Yang, S. Mack, D. D. Awschalom, R. C. Myers, and J. P. Heremans, Phys. Rev. Lett. 106, 186601 (2011). 